\title{
Vandermonde-Type Matrices in Two Step Collocation Methods for Special Second Order Ordinary Differential Equations
}

\author{
Silvana Martucci ${ }^{1}$ and Beatrice Paternoster ${ }^{2}$ \\ 1 Dipartimento di Matematica e Applicazioni, Universitá di Napoli, Italy \\ 2 Dipartimento di Matematica e Informatica, Universitá di Salerno, Italy
}

\begin{abstract}
We consider a general family of two step nonlinear methods for the numerical integration of Ordinary Differential Equations of type $y^{\prime \prime}=f(x, y)$. By applying a collocation technique, linear systems with a Vandermonde-type matrix arise during the construction of the methods. The computation of its determinant reduces to the computation of a recurrence formula depending on the collocation abscissas.
\end{abstract}

\section{Introduction}

We are concerned with the derivation of a general family of two-step collocation methods for the numerical integration of second order Ordinary Differential Equations (ODEs), in which the first derivative does not appear explicitly,

$$
y^{\prime \prime}(t)=f(t, y(t)), \quad y\left(t_{0}\right)=y_{0}, \quad y^{\prime}\left(t_{0}\right)=y_{0}^{\prime}, \quad y(t), f(t, y) \in R^{s} .
$$

The idea behind polynomial collocation is well known and old 13. After fixing a set of collocation abscissas, the numerical solutions is given by a polynomial which satisfies the differential equation at the given set of collocation points, and which reproduces the values already obtained in the previous step point. One-step collocation methods for ODEs (1) form a subset of implicit Runge-Kutta (RK) methods [3] and have been exstensively studied. Multistep collocation methods were first investigated by Guillon and Soulé [2] Then Lie and Norsett [5] considered multistep RK of Gauss type, and Hairer and Wanner [4] those of Radau type. The parameters of the $\mathrm{k}$-step s-stage multistep RK method of Radau type are listed in [6].

We extend the procedure indicated in 4], to obtain a general family of two step collocation methods for (1) within the family of two step Runge-KuttaNyström (TSRKN) methods, introduced in 8,9, providing numerical approximetions not only for the solution, but also to its first derivative at the step point. 


\section{Construction of the Method}

The following definition generalizes the Definition 3.2 of 4], to obtain a general family of two step collocation methods for the ODEs (1):

Definition 1. Let $m$ real numbers $c_{1}, \ldots, c_{m} \in[0,1]$, the solution values $y_{n}, y_{n-1}$ and the derivative values $y_{n}^{\prime}, y_{n-1}^{\prime}$. The collocation polynomial $P(x)$ of degree $2 m+3$ is then defined by:

$$
\begin{gathered}
P\left(x_{n-1}\right)=y_{n-1}, \quad P\left(x_{n}\right)=y_{n}, \quad P^{\prime}\left(x_{n-1}\right)=y_{n-1}^{\prime}, \quad P^{\prime}\left(x_{n}\right)=y_{n}^{\prime}, \\
P^{\prime \prime}\left(x_{n-1}+c_{i} h\right)=f\left(x_{n-1}+c_{i} h, P\left(x_{n-1}+c_{i} h\right)\right), \\
P^{\prime \prime}\left(x_{n}+c_{i} h\right)=f\left(x_{n}+c_{i} h, P\left(x_{n}+c_{i} h\right)\right) .
\end{gathered}
$$

Then the numerical solution of (1) is given by

$$
y_{n+1}=P\left(x_{n+1}\right), \quad y_{n+1}^{\prime}=P^{\prime}\left(x_{n+1}\right)
$$

(2)-(4) constitute a Hermite interpolation problem with incomplete data, because the function values at $x_{n}+c_{i} h$ are missing. Following [46], to compute the collocation polynomial $P(x)$ for $k=2$, we introduce the dimensionless coordinate $t=\left(x-x_{n}\right) / h, x=x_{n}+t h$, with nodes $t_{1}=-1, t_{2}=0$, and define the following polynomials, which constitute a generalized Lagrange basis:

- $\phi_{i}(t), i=1,2$, of degree $2 m+3$, defined by

$$
\begin{gathered}
\phi_{i}\left(t_{j}\right)=\delta_{i j}, \quad \phi_{i}^{\prime}\left(t_{j}\right)=0, \quad i, j=1,2, \\
\phi_{i}^{\prime \prime}\left(c_{j}-1\right)=0, \quad \phi_{i}^{\prime \prime}\left(c_{j}\right)=0, \quad i=1,2, \quad j=1, \ldots, m .
\end{gathered}
$$

- $\psi_{i}(t), i=1,2$, of degree $2 m+3$, defined by

$$
\begin{gathered}
\psi_{i}\left(t_{j}\right)=0, \quad \psi^{\prime}\left(t_{j}\right)=\delta_{i j}, \quad i, j=1,2, \\
\psi_{i}^{\prime \prime}\left(c_{j}-1\right)=0, \quad \psi_{i}^{\prime \prime}\left(c_{j}\right)=0, \quad i=1,2, \quad j=1, \ldots, m .
\end{gathered}
$$

- $\chi_{i, n-1}(t)$ and $\chi_{i, n}(t), i=1, \ldots, m$, of degree $2 m+3$, defined by

$$
\begin{gathered}
\chi_{i, n-1}\left(t_{j}\right)=0, \quad \chi_{i, n}\left(t_{j}\right)=0, \quad i=1, \ldots, m, \quad j=1,2, \\
\chi_{i, n-1}^{\prime}\left(t_{j}\right)=0, \quad \chi_{i, n}^{\prime}\left(t_{j}\right)=0, \quad i=1, \ldots, m, \quad j=1,2, \\
\chi_{i, n-1}^{\prime \prime}\left(c_{j}-1\right)=\delta_{i j}, \quad \chi_{i, n-1}^{\prime \prime}\left(c_{j}\right)=0, \quad i, j=1, \ldots, m, \\
\chi_{i, n}^{\prime \prime}\left(c_{j}-1\right)=0, \quad \chi_{i, n}^{\prime \prime}\left(c_{j}\right)=\delta_{i j}, \quad i, j=1, \ldots, m .
\end{gathered}
$$


$\delta_{i j}$ denotes the Kronecker tensor. Then the expression of the collocation polynomial $P(x)$ in terms of these polynomials is given by:

$$
\begin{aligned}
P\left(x_{n}+t h\right)= & \phi_{1}(t) y_{n-1}+\phi_{2}(t) y_{n}+h\left(\psi_{1}(t) y_{n-1}^{\prime}+\psi_{2}(t) y_{n}^{\prime}\right)+ \\
& h^{2} \sum_{j=1}^{m}\left(\chi_{j, n-1}(t) P^{\prime \prime}\left(x_{n-1}+c_{j} h\right)+\chi_{j, n}(t) P^{\prime \prime}\left(x_{n}+c_{j} h\right)\right) .
\end{aligned}
$$

After constructing $\phi_{i}(t), \psi_{i}(t), \chi_{i, n-1}(t)$ and $\chi_{i, n}(t)$, by putting $t=c_{i}$, writing $P\left(x_{n}+c_{i} h\right)=Y_{n}^{i}$ and inserting the collocation conditions (2)-(4) for $k=2$, we obtain the expression of the TSRKN collocation method, as the following theorem states:

Theorem 1. The collocation method defined by (2)-(3)-(4) is equivalent to the TSRKN method:

$$
\begin{aligned}
Y_{n}^{j}= & u_{j, 1} y_{n-1}+u_{j, 2} y_{n}+h\left(\bar{u}_{j, 1} y_{n-1}^{\prime}+\bar{u}_{j, 2} y_{n}^{\prime}\right)+ \\
& h^{2} \sum_{s=1}^{m}\left(a_{j s} f\left(x_{n-1}+c_{s} h, Y_{n-1}^{s}\right)+b_{j s} f\left(x_{n}+c_{s} h, Y_{n}^{j}\right)\right), \\
y_{n+1}= & \theta_{1} y_{n-1}+\theta_{2} y_{n}+h\left(\eta_{1} y_{n-1}^{\prime}+\eta_{2} y_{n}^{\prime}\right)+ \\
& h^{2} \sum_{j=1}^{m}\left(v_{j} f\left(x_{n-1}+c_{j} h, Y_{n-1}^{j}\right)+w_{j} f\left(x_{n}+c_{j} h, Y_{n}^{j}\right)\right), \\
h y_{n+1}^{\prime}= & \theta_{1}^{\prime} y_{n-1}+\theta_{2}^{\prime} y_{n}+h\left(\eta_{1}^{\prime} y_{n-1}^{\prime}+\eta_{2}^{\prime} y_{n}^{\prime}\right)+ \\
& h^{2} \sum_{j=1}^{m}\left(v_{j}^{\prime} f\left(x_{n-1}+c_{j} h, Y_{n-1}^{j}\right)+w_{j}^{\prime} f\left(x_{n}+c_{j} h, Y_{n}^{j}\right)\right),
\end{aligned}
$$

where

$$
\begin{gathered}
\theta_{i}=\phi_{i}(1), \quad u_{j, i}=\phi_{i}\left(c_{j}\right), \quad i=1,2, j=1, \ldots, m \\
\eta_{i}=\psi_{i}(1), \quad \bar{u}_{j, i}=\psi_{i}\left(c_{j}\right), \quad i=1,2, j=1, \ldots, m \\
v_{j}=\chi_{j, n-1}(1), \quad a_{j s}=\chi_{j, n-1}\left(c_{s}\right), \quad j, s,=1, \ldots, m, \quad j, s,=1, \ldots, m \\
w_{j}=\chi_{j, n}(1), \quad b_{j s}=\chi_{j, n}\left(c_{s}\right), \quad j=1,2, \\
\theta_{i}^{\prime}=\phi_{i}^{\prime}(1), \quad \eta_{i}^{\prime}=\psi_{i}^{\prime}(1), \quad i=
\end{gathered}
$$




$$
v_{j}^{\prime}=\chi_{j, n-1}^{\prime}(1), \quad w_{j}^{\prime}=\chi_{j, n}^{\prime}(1), \quad j,=1, \ldots, m
$$

and $\phi_{i}(t), \psi_{i}(t), \chi_{i, n-1}(t)$ and $\chi_{i, n}(t)$ are the polynomials defined by the conditions (6)-(13).

Let us note that the order of approximation of $P\left(x_{n}+c_{i} h\right)$, that is the so called stage order of the TSRKN method, is $2 m+3$.

Let us now show how to construct the polynomials $\phi_{i}(t), \psi_{i}(t), \chi_{i, n-1}(t)$ and $\chi_{i, n}(t)$. We will follow the procedure indicate in [6]. We expand the polynomials:

$$
\begin{gathered}
\phi_{i}(t)=\sum_{k=0}^{2 m+3} d_{k}^{(i)} t^{k}, \quad \psi_{i}(t)=\sum_{k=0}^{2 m+3} e_{k}^{(i)} t^{k}, \quad i=1,2, \\
\chi_{i, n-1}(t)=\sum_{k=0}^{2 m+3} p_{k}^{(i)} t^{k}, \quad \chi_{i, n}(t)=\sum_{k=0}^{2 m+3} q_{k}^{(i)} t^{k}, \quad i=1, \ldots m .
\end{gathered}
$$

The following linear systems arise: $H d^{(i)}=N_{0}^{(i)}, H e^{(i)}=N_{1}^{(i)}, H p^{(i)}=N_{2, n-1}^{(i)}$ and $H q^{(i)}=N_{2, n}^{(i)}$, of order $2 m+4$ with

$$
H=\left(\begin{array}{cccccc}
1 & t_{1} & t_{1}^{2} & t_{1}^{3} & \ldots & t_{1}^{2 m+3} \\
1 & t_{2} & t_{2}^{2} & t_{2}^{3} & \ldots & t_{2}^{2 m+3} \\
0 & 1 & 2 t_{1} & 3 t_{1}^{2} & \ldots & (2 m+3) t_{1}^{2 m+2} \\
0 & 1 & 2 t_{2} & 3 t_{2}^{2} & \ldots & (2 m+3) t_{2}^{2 m+2} \\
0 & 0 & 2 & 2 \cdot 3\left(c_{1}-1\right) & \ldots & (2 m+2)(2 m+3)\left(c_{1}-1\right)^{2 m+1} \\
\vdots & \vdots & & & \vdots & \vdots \\
0 & 0 & 2 & 2 \cdot 3\left(c_{m}-1\right) & \ldots & (2 m+2)(2 m+3)\left(c_{m}-1\right)^{2 m+1} \\
0 & 0 & 2 & 2 \cdot 3 c_{1} & \ldots & (2 m+2)(2 m+3) c_{1}^{2 m+1} \\
\vdots & \vdots & & & \vdots & \vdots \\
0 & 0 & 2 & 2 \cdot 3 c_{m} & \ldots & (2 m+2)(2 m+3) c_{m}^{2 m+1}
\end{array}\right)
$$

$$
d^{(i)}=\left(\begin{array}{c}
d_{0}^{(i)} \\
d_{1}^{(i)} \\
d_{2}^{(i)} \\
d_{3}^{(i)} \\
d_{4}^{(i)} \\
\vdots \\
\vdots \\
d_{2 m+3}^{(i)}
\end{array}\right), e^{(i)}=\left(\begin{array}{c}
e_{0}^{(i)} \\
e_{1}^{(i)} \\
e_{2}^{(i)} \\
e_{3}^{(i)} \\
e_{4}^{(i)} \\
\vdots \\
\vdots \\
e_{2 m+3}^{(i)}
\end{array}\right), p^{(i)}=\left(\begin{array}{c}
p_{0}^{(i)} \\
p_{1}^{(i)} \\
p_{2}^{(i)} \\
p_{3}^{(i)} \\
p_{4}^{(i)} \\
\vdots \\
\vdots \\
p_{2 m+3}^{(i)}
\end{array}\right), q^{(i)}=\left(\begin{array}{c}
q_{0}^{(i)} \\
q_{1}^{(i)} \\
q_{2}^{(i)} \\
q_{3}^{(i)} \\
q_{4}^{(i)} \\
\vdots \\
\vdots \\
q_{2 m+3}^{(i)}
\end{array}\right),
$$




$$
N_{0}^{(i)}=\left(\begin{array}{c}
\delta_{i 1} \\
\delta_{i 2} \\
0 \\
0 \\
0 \\
\vdots \\
0 \\
0 \\
\vdots \\
0
\end{array}\right) \quad N_{1}^{(i)}=\left(\begin{array}{c}
0 \\
0 \\
\delta_{i 1} \\
\delta_{i 2} \\
0 \\
\vdots \\
0 \\
0 \\
\vdots \\
0
\end{array}\right) \quad N_{2, n-1}^{(i)}=\left(\begin{array}{c}
0 \\
0 \\
0 \\
0 \\
\delta_{i 1} \\
\vdots \\
\delta_{i m} \\
0 \\
\vdots \\
0
\end{array}\right) \quad N_{2, n}^{(i)}=\left(\begin{array}{c}
0 \\
0 \\
0 \\
0 \\
0 \\
\vdots \\
0 \\
\delta_{i 1} \\
\vdots \\
\delta_{i m}
\end{array}\right) .
$$

To compute the coefficients of the collocation method defined in Theorem 1 , we must now evaluate the polynomials $\phi_{i}(t), \psi_{i}(t), \chi_{i, n-1}(t)$ and $\chi_{i, n}(t)$ according to (14)-(19). When $H$ is not singular, the computation yields

$$
\begin{aligned}
& \phi_{i}(t)=\left[1 t \ldots t^{2 m+3}\right] H^{-1} N_{0}^{(i)}, \quad i=1,2 \\
& \theta_{i}=\phi_{i}(1)=\left[\begin{array}{lll}
1 & \ldots & 1
\end{array}\right] H^{-1} N_{0}^{(i)} \text {, } \\
& u_{j i}=\phi_{i}\left(c_{j}\right)=\left[1 c_{j} \ldots c_{j}^{2 m+3}\right] H^{-1} N_{0}^{(i)}, i=1,2, \quad j=1, \ldots, m \\
& \psi_{i}(t)=\left[1 t \ldots t^{2 m+3}\right] H^{-1} N_{1}^{(i)}, \quad i=1,2 \\
& \eta_{i}=\psi_{i}(1)=\left[\begin{array}{lll}
1 & \ldots & 1
\end{array}\right] H^{-1} N_{1}^{(i)}, \\
& \bar{u}_{j i}=\psi_{i}\left(c_{j}\right)=\left[1 c_{j} \ldots c_{j}^{2 m+3}\right] H^{-1} N_{1}^{(i)}, i=1,2, \quad j=1, \ldots, m \\
& \chi_{i, n-1}(t)=\left[1 t \ldots t^{2 m+3}\right] H^{-1} N_{2, n-1}^{(i)}, \quad i=1, \ldots, m \\
& v_{i}=\chi_{i, n-1}(1)=\left[\begin{array}{lll}
1 & \ldots & 1
\end{array}\right] H^{-1} N_{2, n-1}^{(i)}, \quad i=1, \ldots, m \\
& a_{i s}=\chi_{i, n-1}\left(c_{s}\right)=\left[\begin{array}{lll}
1 & c_{s} \ldots c_{s}^{2 m+3}
\end{array}\right] H^{-1} N_{2, n-1}^{(i)}, \quad i, s=1, \ldots, m \\
& \chi_{i, n}(t)=\left[1 t \ldots t^{2 m+3}\right] H^{-1} N_{2, n}^{(i)}, \quad i=1, \ldots, m \\
& v_{i}=\chi_{i, n}(1)=\left[\begin{array}{lll}
1 & \ldots & 1
\end{array}\right] H^{-1} N_{2, n}^{(i)}, \quad i=1, \ldots, m \\
& b_{i s}=\chi_{i, n}\left(c_{s}\right)=\left[\begin{array}{lll}
1 & c_{s} \ldots c_{s}^{2 m+3}
\end{array}\right] H^{-1} N_{2, n}^{(i)}, \quad i, s=1, \ldots, m .
\end{aligned}
$$

For $m \geq 2$, from the initial and collocation conditions (2)-(4), a linear system of $2 m+4$ equations in $2 m+4$ unknowns arises, having $H$ in (20) as matrix of coefficients, where $t_{1}=-1$ and $t_{2}=0$.

The computation of the determinant of $H$ allows us to determine the exceptional values of the collocation abscissas $c_{i}$ for which the two step collocation method cannot be constructed.

\section{Computation of the Determinant of $\mathbf{H}$}

The determinant of $H$ is computed through the tecnique which is usually applied to the Vandermonde matrices; in this way the computation of the determinant of $\mathrm{H}$ reduces to the computation of a $2 \times 2$ matrix, whose elements are defined through a recurrence formula, as described in the following.

Let us reduce the determinant of $H$ to the determinant of the $(2 m+2) \times$ $(2 m+2)$ matrix $H^{\prime}$, that is $\operatorname{det}(H)=-\operatorname{det}\left(H^{\prime}\right)$, where 


$$
H^{\prime}=\left(\begin{array}{cccc}
1 & -1 & \ldots & (-1)^{2 m+3} \\
-2 & 3 & \ldots & (2 m+3)(-1)^{2 m+2} \\
2 & 2 \cdot 3\left(c_{1}-1\right) & \ldots & (2 m+2)(2 m+3)\left(c_{1}-1\right)^{2 m+1} \\
\vdots & \vdots & \vdots & \vdots \\
2 & 2 \cdot 3\left(c_{m}-1\right) & \ldots & (2 m+2)(2 m+3)\left(c_{m}-1\right)^{2 m+1} \\
2 & 2 \cdot 3 c_{1} & \ldots & (2 m+2)(2 m+3) c_{1}^{2 m+1} \\
\vdots & \vdots & \vdots & \vdots \\
2 & 2 \cdot 3 c_{m} & \ldots & (2 m+2)(2 m+3) c_{m}^{2 m+1}
\end{array}\right)
$$

Then $\operatorname{det}(H)=-(2 m+2) !(2 m+3) ! \operatorname{det} H^{\prime \prime}$,

$$
H^{\prime \prime}=\left(\begin{array}{cccc}
\frac{1}{2} & (-1) \frac{1}{2 \cdot 3} & \cdots & \frac{1}{(2 m+2)(2 m+3)}(-1)^{2 m+1} \\
-1 & \frac{1}{2} & \ldots & \frac{-1}{2 m+3}(-1)^{2 m+1} \\
1 & \left(c_{1}-1\right) & \ldots & \left(c_{1}-1\right)^{2 m+1} \\
\vdots & \vdots & \vdots & \vdots \\
1 & \left(c_{m}-1\right) & \ldots & \left(c_{m}-1\right)^{2 m+1} \\
1 & c_{1} & \ldots & c_{1}^{2 m+1} \\
\vdots & \vdots & \vdots & \vdots \\
1 & c_{m} & \ldots & c_{m}^{2 m+1}
\end{array}\right) .
$$

By setting

$$
\begin{gathered}
x_{1}=c_{1}-1, \ldots, x_{m}=c_{m}-1, x_{m+1}=c_{1}, \ldots, x_{2 m}=c_{m}, \\
r^{(0)}(n)=\frac{1}{n(n+1)}(-1)^{n+1}, \quad q^{(0)}(n)=\frac{-1}{n}(-1)^{n+1},
\end{gathered}
$$

the matrix $H^{\prime \prime}$ can be written as

$$
H^{\prime \prime}=\left(\begin{array}{cccc}
r^{(0)}(1) & r^{(0)}(2) & \ldots & r^{(0)}(n) \\
q^{(0)}(1) & q^{(0)}(2) & \ldots & q^{(0)}(n) \\
1 & x_{1} & \ldots & x_{1}^{2 m+1} \\
\vdots & \vdots & \vdots & \vdots \\
1 & x_{m} & \ldots & x_{m}^{2 m+1} \\
1 & x_{m+1} & \ldots & x_{m+1}^{2 m+1} \\
\vdots & \vdots & \vdots & \vdots \\
1 & x_{2 m} & \ldots & x_{2 m}^{2 m+1}
\end{array}\right)
$$

Then $H^{\prime \prime}$ is a Vandermonde matrix except the firt two rows. Its determinant is now computed by using the tecnique which is usually applied to the Vandermonde determinant. In details, we multiply each column by $x_{1}$ and subtract it from the following column, starting from the penultimate column. At the end a block matrix is obtained: 


$$
H^{\prime \prime}=\prod_{i<j, i=1}^{2 m}\left(x_{i}-x_{j}\right)\left(\begin{array}{cc}
A & B \\
V & 0
\end{array}\right)
$$

where $A$ is a $2 \times m$ matrix, 0 is the zero matrix of dimension $2 m \times 2, V$ is a lower triangular matrix with 1 on the diagonal, and $B$ is a $2 \times 2$ matrix containing the elements of $H^{\prime \prime}$ in the upper right corner, which result after the transformations performed on $H^{\prime \prime}$ to compute its determinant. In particular $B$ takes the following form:

$$
B=\left(\begin{array}{ll}
r^{(2 m)}(2 m+1) & r^{(2 m)}(2 m+2) \\
q^{(2 m)}(2 m+1) & q^{(2 m)}(2 m+2)
\end{array}\right)
$$

where the $r$ and $q$ elements in B are derived through the following recurrence formulas, which keep track of the trasformation on $H^{\prime \prime}$ :

$$
\begin{gathered}
r^{(0)}(n)=\frac{1}{n(n+1)}(-1)^{n+1}, \quad r^{(i)}(n)=r^{(i-1)}(n)-r^{(i-1)}(n-1) x_{i}, \\
q^{(0)}(n)=\frac{-1}{n}(-1)^{n+1}, \quad q^{(i)}(n)=q^{(i-1)}(n)-q^{(i-1)}(n-1) x_{i}, \\
i=1, \ldots, 2 m, n=1, \ldots, 2 m .
\end{gathered}
$$

By considering the elementary symmetric polynomials in $x_{1}, \ldots, x_{2 m}$,

$$
p_{1}=\sum x_{i}, \quad p_{2}=\sum_{i<j} x_{i} x_{j}, \quad p_{s}=\sum_{i_{1}<i_{2}<i_{s}} x_{i_{1}} x_{i_{2}} \ldots x_{i_{s}},
$$

it is possible to prove by induction that

$$
r^{(j)}(n+1)=\sum_{k=0}^{j}(-1)^{k} r^{(0)}(n-k) p_{k}, \quad q^{(j)}(n+1)=\sum_{k=0}^{j}(-1)^{k} q^{(0)}(n-k) p_{k} .
$$

In conclusion

$$
\operatorname{det}(H)=-(2 m+2) !(2 m+3) ! \prod_{i<j, i=1}^{2 m}\left(x_{j}-x_{i}\right) \operatorname{det}(B)
$$

where

$$
\operatorname{det}(B)=r^{(2 m)}(2 m+1) q^{(2 m)}(2 m+2)-q^{(2 m)}(2 m+1) r^{(2 m)}(2 m+2)
$$

In this way also the computation of the exceptional values of the collocation abscissas for the method defined in theorem 1 reduces to the computation of the $c$-values which annihilate the $2 \times 2$ determinant of $B$ in (21). 
For instance, the computation of $\operatorname{det}(\mathrm{H})$ for $m=1$ allows us to state that the collocation-based TSRKN is defined only if $c \neq \pm \sqrt{\frac{3}{10} \pm \frac{\sqrt{\frac{11}{6}}}{5}}$.

The resulting expressions for a higher number of stages can be easily derived through the illustrated technique and the usage of symbolic computation.

\section{Concluding Remark}

In this paper the coefficients of the new two step collocation methods for ODEs (1) are defined through the computation of the inverse of matrix $H$ in (20), which is of Vandermonde type. Different approaches for the derivation of collocationbased TSRKN methods are under consideration, following 4|5|7], that avoid the numerical treatment of Vandermonde-type matrices, involving on the contrary the integrals of polynomials in the generalized Lagrange basis. The analysis of the properties of the TSRKN methods, such as the obtainable order of convergence, stability properties, efficiency and so on, will be subject of a forthcoming paper.

\section{References}

1. J. C. Butcher, The Numerical Analysis of Ordinary Differential Equations: RungeKutta and General Linear Methods, Wiley, Chichester, 1987.

2. A. Guillon and J. L. Soulé, La résolution numérique des problémes différentiels aux conditions initiales par des méthodes de collocation, RAIRO Anal. Numér. Ser. Rouge R-3, 17-44, 1969.

3. E. Hairer, S. P. Norsett and G. Wanner, Solving Ordinary Differential Equations I - Nonstiff Problems, Springer Series in Computational Mathematics 8, SpringerVerlag, Berlin, 1987.

4. E. Hairer and G. Wanner, Solving Ordinary Differential Equations II: Stiff and Differential-Algebraic Problems, Springer, Berlin, 1991.

5. I. Lie and S. P. Norsett, Superconvergence for multistep collocation, Math. Comput. 52, 65-79, 1989.

6. E. Messina, J. J. B. de Swart and W. van der Veen, Parallel iterative linear solvers for multistep Runge-Kutta methods, J. Comput. Appl. Math. 85, 145-167, 1997.

7. B. Paternoster, General two-step Runge-Kutta methods based on algebraic and trigonometric polynomials, Int. J. Appl. Math. 6(4), 347-362, 2001.

8. B. Paternoster, Two step Runge-Kutta-Nyström methods for $y^{\prime \prime}=f(x, y)$ and Pstability, Computational Science - ICCS 2002, Lecture Notes in Computer Science 2331, Part III, P.M.A.Sloot, C.J.K.Tan, J.J.Dongarra, A.G.Hoekstra Eds., 459466, Springer Verlag, Amsterdam (2002).

9. B.Paternoster, Two step Runge-Kutta-Nystrom methods for oscillatory problems based on mixed polynomials, Computational Science - ICCS 2003, Lecture Notes in Computer Science 2658, Part II, P.M.A.Sloot, D.Abramson, A.V.Bogdanov, J.J.Dongarra, A.Y.Zomaya, Y.E.Gorbachev Eds., pp. 131-138, Springer, Berlin Heidelberg (2003). 